\title{
Brustrekonstruktion mit freien TRAM oder DIEP Lappen - Was ist zeitgemäßer Standard?
}

\author{
Konsensuspapier der Deutschen Arbeitsgemeinschaft für \\ Mikrochirurgie der peripheren Nerven und Gefäße
}

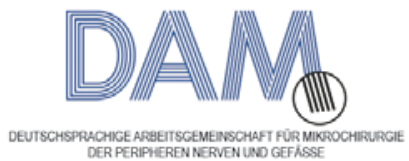

\section{Breast reconstruction with the free TRAM or DIEP flap - What is the current standard?}

\author{
Consensus Statement of the German Speaking Working Group for \\ Microsurgery of the Peripheral Nerves and Vessels
}

\footnotetext{
Autoren

Institut

1 Universitätsklinikum Freiburg, Klinik für Plastische und Handchirurgie.

2 Stanford University Medical Center, Stanford, CA, Division of Plastic and Reconstructive Surgery.

3 Helios-Klinikum Emil von Behring, Berlin, Klinik für Plastische und Ästhetische Chirurgie.

4 Universitätsklinikum Erlangen, Plastisch- und Handchirurgische Klinik.

5 Abteilung für Plastische, Rekonstruktive und Ästhetische Chirurgie, Ospedale Regionale di Lugano (ORL), Ente Ospedaliero Cantonale (EOC), Lugano, Schweiz.

6 Praxis Heitmann \& Fansa, Ästhetisch Plastische Chirurgie, München.

7 Klinikum St. Georg Leipzig, Klinik für Plastische und Handchirurgie mit Schwerbrandverletztenzentrum.

8 Agaplesion Markus Krankenhaus, Klinik für Plastische und Ästhetische Chirurgie, Wiederherstellungs- und Handchirurgie.

9 BG Unfallklinik Ludwigshafen, Klinik für Hand, Plastische und Rekonstruktive Chirurgie, Schwerbrandverletztenzentrum.
}

Steffen U. Eisenhardt' ${ }^{1}$, Arash Momeni², Uwe von Fritschen ${ }^{3}$, Raymund E. Horch ${ }^{4}$, G.Björn Stark ${ }^{1}$, Holger Bannasch ${ }^{1}$, Yves Harder $^{5}$, Christoph Heitmann ${ }^{6}$, Thomas Kremer ${ }^{7}$, Ulrich M. Rieger ${ }^{8}$, Ulrich Kneser ${ }^{9}$

Schlüsselwörter

Mammarekonstruktion, Brustchirurgie, freie Lappenplastiken, Mikrochirurgie, Perforans-Lappenplastiken, Mamma, Körperregion und Anatomie, TRAM Lappen, DIEP Lappen

Key words

breast reconstruction, free flap grafts, perforator flap grafts, breast, TRAM flap, DIEP flap

eingereicht 22.02.2018

akzeptiert 27.04.2018
Bibliografie

DOI https://doi.org/10.1055/a-0631-9025

Handchir Mikrochir Plast Chir 2018; 50: 248-255

(c) Georg Thieme Verlag KG Stuttgart · New York

ISSN 0722-1819

Korrespondenzadresse

Univ.-Prof. Dr. med. Steffen U. Eisenhardt

Sektion für Rekonstruktive Mikrochirurgie

Klinik für Plastische und Handchirurgie

Universitätsklinikum Freiburg

Hugstetter Strasse 55

79106 Freiburg

Telefax: +49 $761270-25010$

E-Mail: steffen.eisenhardt@uniklinik-freiburg.de

\section{ZUSAMMENFASSUNG}

Die Brustrekonstruktion mit freiem Gewebetransfer vom Unterbauch als (muskelsparende) TRAM oder DIEP Lappenplastik stellt das Standardverfahren der autologen Brustrekonstruktion dar. Durch operative Fortschritte ist es gelungen die Hebedefektmorbidität durch muskelsparende bzw. perforatorbasierte Techniken zu minimieren. Hier stellt sich die Frage, in wie weit dies als ein notwendiger Standard angesehen werden kann oder ob auch Lappenplastiken mit Muskelanteilen (MS-TRAM) noch als zeitgemäß anzusehen sind. In einem Konsensusworkshop der 3 Jahrestagung der Deutschsprachigen Arbeitsgemeinschaft für Mikrochirurgie der peripheren Nerven und Gefäße (DAM) wurde die Datenlage kritisch geprüft und durch eine Expertengruppe ein Konsensus erarbeitet. Dieser beinhaltet neben den aktuellen Daten zu Lappensicherheit und Hebedefektmorbidität aus der Literatur auch ein aktuelles Meinungsbild der Expertengruppe bzgl. verschiedener technischer Details des operativen Vorgehens.
Telefon: +49 $761270-25730$ 


\section{ABSTRACT}

The most common donor-site for autologous breast reconstruction is the abdomen. Over the past several decades technical advances have resulted in the development of flaps that have been associated with a progressive decrease in abdominal wall morbidity. However, controversy exists related to the differences between muscle-sparing (MS)TRAM and deep inferior epigastric perforator (DIEP) flaps. Hence, the question which approach should be considered standard of care remains unanswered. To address this question the current literature and published evidence was critically reviewed and discussed by an expert panel at the 39th Annual Meeting of the German-speaking Society for Micro surgery of the Peripheral Nerves and Vessels (DAM). Based on this discussion a consensus statement was developed that incorporates contemporary data regarding postoperative complication rate, donor site morbidity, as well as expert opinion regarding technical details in autologous breast reconstruction with free TRAM and DIEP flaps

\section{Einleitung}

Die autologe Brustrekonstruktion durch Gewebe vom Unterbauch hat sich nach ihrer Beschreibung zu Beginn der 80 er Jahre schnell zu einem der Standardverfahren in der rekonstruktiven Mikrochirurgie entwickelt $[1,2]$.

In klinischen Nachuntersuchungen hat sich der Vorteil des freien Gewebetransfers gegenüber dem gestielten TRAM Lappen bestätigt. Dies ist von der aktuellen Studienlage gut belegt. Insbesondere die Hebedefektmorbidität des gestielten TRAM Lappens hat sich auch in größeren Übersichtsarbeiten gegenüber dem DIEP Lappen als nicht konkurrenzfähig gezeigt [3]. Auch die Rate der Fettgewebsnekrosen und Lappenteilverluste ist bei freien TRAM Lappen gegenüber dem gestielten TRAM Lappen reduziert [4]. Insofern muss der gestielte TRAM Lappen zur Brustrekonstruktion, der in den USA immer noch häufig verwendet wird, kritisch gesehen werden.

Nach der Erstbeschreibung der Hebung eines freien Lappens vom Unterbauch ohne Muskelanteile wurde der DIEP Lappen rasch als Goldstandard zur autologen Brustrekonstruktion propagiert [5, 6] (- Abb. 1). Dies beruht auf der Annahme, dass durch die Schonung der Muskulatur die Hebedefektmorbidität reduziert wird. In einer Expertenrunde wurde daher im Rahmen des DAM Konsensusworkshops auf der Jahrestagung der Deutschsprachigen Arbeitsgemeinschaft für Mikrochirurgie der peripheren Nerven und Gefäße (DAM) 2017 in Freiburg im Breisgau die aktuelle Datenlage zur Belegung dieser Annahme diskutiert, ebenso wie die aktuellen Daten zur Sicherheit der Lappendurchblutung. Letzteres vor dem Hintergrund, dass die ausgedehntere und selektivere Präparation des DIEP Lappens mit einer erhöhten Komplikationsrate der Lappenplastik per se einhergehen kann.

\section{Methode}

Der dargestellte Konsens wurde im Rahmen des Konsensusworkshops der 39. Jahrestagung der Deutschsprachigen Arbeitsgemeinschaft für Mikrochirurgie der peripheren Nerven und Gefäße (DAM) in Freiburg im Breisgau durch ein Expertengremium erstellt. Die verfassten Empfehlungen basieren auf der aktuellen Literaturlage und der Expertenmeinung der Teilnehmer dieses Workshops. Es handelt sich daher nicht um eine evidenzbasierte Leitlinie.
- Tab. 1 Klassifikation der freien TRAM Lappenplastiken modifiziert nach Nahabedian [7] orientierend an den in den Lappen miteinbezogenen $\mathrm{M}$. rectus abdominis Anteilen.

\begin{tabular}{|l|l|}
\hline Nomenklatur & Definition (M. rectus abdominis Anteil) \\
\hline MS-0 & Segmentale Hebung in voller Breite \\
\hline MS-1 & Belassung eines lateralen Muskelanteils \\
\hline MS-2 & Belassung eines lateralen und medialen Muskelanteils \\
\hline MS-3 (DIEP) & Belassung des gesamten Muskels \\
\hline
\end{tabular}

\section{Definition des MS-TRAM und DIEP Lappens}

\section{Muscle-sparing free transverse rectus abdominis myocutaneous (MS-TRAM)}

Sowohl MS-TRAM als auch der DIEP Lappen beruhen auf dem tiefen inferioren epigastrischen Gefäßsystem. Zur Präparation des Gefäßstiels wird bei beiden die vordere Rektusscheide eröffnet und der M. rectus abdominis entlang des Faserverlaufes gespalten. Bei der Präparation des MS-TRAM wird lediglich ein Teil des Muskels in den Lappen inkorporiert, wohingegen der DIEP Lappen, per Definition, keine Muskelanteile enthält. Die gängige Subklassifikation der MS-TRAM Lappenplastiken beruht auf der Arbeit von Nahabedian et al [7]. Hierin wird die Hebung eines Segmentes des M. rectus abdominis in voller Breite als MS-0, die Schonung eines lateralen Muskelanteils als MS-1, das Belassen sowohl lateraler als auch medialer Muskelanteile als MS-2 und die Lappenhebung ohne Muskeleinschluss als MS-3 TRAM bezeichnet ( $\triangleright$ Tab. 1). Das zugrundeliegende Konzept basiert auf der Annahme, dass durch zunehmende Schonung der Muskelanteile die Integrität der Bauchwand bestehen bleibt. Zum anderen soll die Innervation des Rektusmuskels erhalten bleiben, welcher segmental über lateral eintretende Interkostalnerven innerviert wird.

Die Möglichkeit bei der Lappenhebung eine größere Anzahl myokutaner Perforatoren einzuschließen, wird im Allgemeinen als Hauptvorteil des MS-TRAM Lappens angegeben.

\section{Deep inferior epigastric perforator (DIEP)}

Im Gegensatz zum TRAM wird der DIEP Lappen über Perforatoren versorgt, die komplett aus dem Muskel herauspräpariert werden. Somit verbleibt weder ein Muskel- noch Fasziendefekt. Die Länge 

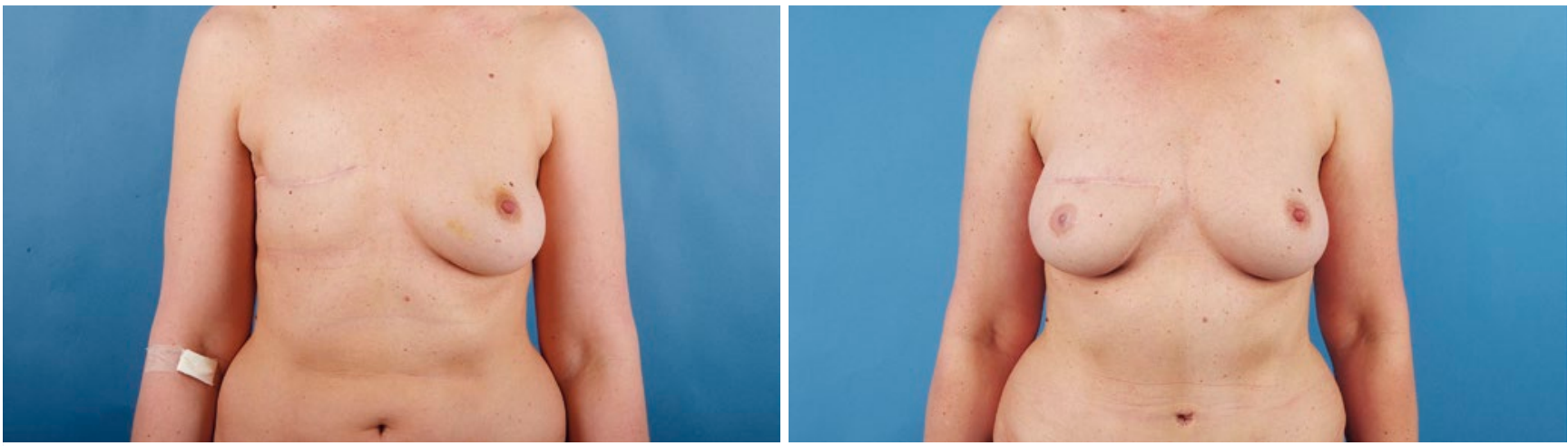

Abb. 1 Brustrekonstruktion mit DIEP-Lappenplastik bei Z. n. Ablatio mammae bei ausgedehntem DCIS. Präoperativ links und 2 Jahre postoperativ rechts.

der intramuskulären Präparation ist jedoch mit einer progressiven Schwächung des Muskels vergesellschaftet, so dass die Präparation multipler Perforatoren über weite Strecken den funktionellen Benefit des DIEP Lappens in Frage stellt. Dabei ist die Auswahl der Perforatoren bzgl. Größe, Lokalisation (mediale oder laterale Perforatoren Reihe), und Verteilung erfahrungsabhängig und begründet die größere Lernkurve dieser Lappenplastik [8]. Das Ziel bei der DIEP Lappenhebung ist es, die intramuskuläre Präparation gering zu halten und möglichst den Rektusmuskel nicht zu denervieren.

Diese theoretische Reduktion der Hebedefektmorbidität wird daher auch als der größte Vorteil dieses Verfahrens gegenüber dem TRAM-Lappen postuliert.

\section{Definition und Problematik im Rahmen der Konsensfindung}

Bei der Gegenüberstellung des MS-TRAM vs DIEP Lappens muss zunächst klar diese Definition eingehalten werden, da auf Grund der teilweise sehr kleinen Muskelsegmente bei MS-2 TRAM Lappenplastiken die Grenzen mitunter unscharf sind. Dies erschwert die Interpretation publizierter Daten. Im Rahmen der Konsensfindung wurde daher entsprechend der obigen Klassifikation der DIEP Lappen als Lappen ohne makroskopische Muskelanteile definiert. Die Definition der MS-0 bis MS-3 TRAM Lappenplastiken orientiert sich an der Definition von Nahabedian ( $\$$ Tab. 1) [7]. Dabei ist die MS-3 TRAM Lappenplastik gleichzusetzen mit der hier verwendeten Definition der DIEP Lappenplastik.

Einschränkend muss angemerkt werden, dass die Klassifikation nach Nahabedian keine Aussage über die Funktionalität der geschonten Muskelanteile zulässt. Insofern kann im Rahmen der DIEP Lappenhebung eine Durchtrennung sämtlicher segmentaler Interkostalnerven zum Rektusmuskel erfolgen, wodurch der funktionelle Vorteil des DIEP-Lappens zunichtegemacht wird. [9-11] Im Gegensatz hierzu kann ein unter Schonung der Interkostalnerven gehobener MS-TRAM mit einer weitgehend erhaltenen Bauchwandintegrität vergesellschaftet sein. Hier zeichnet sich bereits eine gewisse Unschärfe ab, die auch die Interpretation der aktuellen Datenlage erschwert. Die gängige MS-Klassifikation bietet hier Raum für Unsicherheiten bzgl. der Funktion des belassenen Muskels. Ferner wird keine Aussage zum Ausmaß des Fasziendefektes getroffen. Somit ist auch eine erhebliche Varianz in der Hebedefektmorbidität zwischen in der MS-Klassifikation als gleichwertig beschriebenen Muskellappenplastiken gegeben und vergleichende Metaanalysen deutlich erschwert. Neben der Nervenschonung sind hier noch die Länge der intramuskulären Präparation, die fasziensparende Lappenhebung und auch technische Details, wie die Verschlusstechnik der vorderen Rektusscheide als entscheidende Faktoren zu nennen, die wesentlich die Hebedefektmorbidität beeinflussen. Diese Faktoren sind Operateur-abhängig und unterliegen keinem allgemeinen Standard.

\section{Aktuelle Datenlage}

Fast 30 Jahre nach Erstbeschreibung der DIEP-Lappenplastik existiert eine Vielzahl an vergleichenden Studien zur Sicherheit und Komplikationsrate nach freien DIEP und freien TRAM-Lappen zur Brustrekonstruktion (mit den o.g. Einschränkungen). Es lassen sich zwei wesentliche zu beurteilende Kriterien festlegen. Zum einen die Perfusionssicherheit des freien TRAM vs. DIEP Lappen in Bezug auf Lappenverluste, Lappenteilverluste und der Rate an Fettgewebsnekrosen. Zum anderen die Hebedefektmorbidität der zu vergleichenden Lappenplastiken, also Bauchwandschwäche, Hernien und „Bulging“.

\section{Lappenüberleben und Fettgewebsnekrosen}

Es existiert eine große Anzahl vergleichender wissenschaftlicher Studien bzgl. Lappenverlusten, Lappenteilverlusten und Fettegewebsnekrosen zwischen DIEP und MS-TRAM Lappenplastiken. Allen gemein sind Schwächen im Studiendesign und die dadurch bedingte eingeschränkte Validität der Schlussfolgerungen. Auf Boden der aktuellen, vorwiegend retrospektiven Arbeiten lassen sich für den MS-TRAM Lappen in der Literatur Lappenverlustraten von $0,3 \%$ bis 3,6\% finden. Diese Zahl ist für DIEP Lappenplastik mit $5 \%$ bis $6 \%$ leicht erhöht. Für Lappenteilverluste werden diese Daten für den MS-TRAM von 2,2\% bis $7 \%$, und für den DIEP Lappen von $2,5 \%$ bis $8 \%$ beziffert [7, 12, 13, 14-22] ( Tab. 2). Die aktuelle Literatur scheint damit eine leicht erhöhte Durchblutungssicherheit für den TRAM Lappen zu belegen, auch wenn einzelne „single-surgeon“ Arbeiten hier abweichende Daten publizieren. So sieht Nahabedian keinen signifikanten Unterschied in seinem Kollektiv zwischen 
\Tab. 2 Tabellarische Zusammenfassung der aktuellen Literatur bzgl. der erhobenen Parameter zur Lappensicherheit und Hebedefektmorbidität. Modifiziert nach Butler et al. [28]

Untersuchter Parameter
Lappenverlust
Fettnekrosen
Bulging/Unterbauchkontur bei unilateraler Lappenhebung
Bulging/Unterbauchkontur bei bilateraler Lappenhebung
Hernienbildung (unilateral)
Hernienbildung (bilateral)

den beiden Verfahren in puncto durchblutungsassoziierte Komplikationsrate [12]. Nelson und Mitarbeiter sahen zwar keine wesentlichen Unterschiede in sogenannten minor-Komplikationen, im Bezug zu Major-Komplikation, inklusive Lappenverlust jedoch einen signifikanten Unterschied von 3,9\% in der DIEP Gruppe vs. $0 \%$ in der TRAM Gruppe bei einem Signifikanzlevel von $p=0,03$ [14]. Hiervon abzugrenzen sind Fettnekrosen, die im Zusammenhang mit additiven Maßnahmen auftreten [23, 24].

Jedoch zeigt sich hier in der aktuellen Literatur eine Variabilität der Komplikationsrate auch abhängig von der Perforatorenwahl. Kamali und Mitarbeiter konnten kürzlich zeigen, dass die laterale Perforatorenreihe generell mit einer verringerten Fettgewebsnekroserate vergesellschaftet ist. Dazu untersuchten die Autoren eine retrospektive Serie von 728 hemi-DIEP Lappen, die für bilaterale Brustrekonstruktionen genutzt wurden. Davon waren eine vergleichbare Anzahl an der medialen Perforatorreihe ( $n=225 ; 30,9 \%)$ und an der lateralen Reihe $(n=225 ; 30,9 \%)$ gestielt. Die verbleibenden Lappen waren mit jeweils medialen und lateralen Perforatoren versorgt ( $n=240 ; 33,0 \%$ ). Hier zeigte sich, dass die Rate der Fettgewebsnekrose bei medialen Perforatoren bei $24,5 \%$ lag und damit signifikant erhöht $(p<0,001)$ zu den Lappen mit lateraler Perforatorversorgung $(8,2 \%)$ war. Ein signifikanter Unterschied zwischen Lappenplastiken mit Perforatoren aus der medialen und lateralen Reihe und lateraler Perforatorversorgung alleine zeigte sich in dieser Serie nicht [25]. Die zunehmende Anzahl an eingeschlossenen Perforatoren korrelierte ferner negativ mit dem Risiko für Fettgewebsnekrosen. Dies ist auch in der prospektiven Arbeit von Baumann und Mitarbeitern belegt [26]. Diese Daten belegen vereinfacht gesagt, dass die Perfusion mit der Anzahl der Perforatoren besser wird und damit die Komplikationsrate sinkt. Dies ist natürlich auch durch das Lappengewicht mitbestimmt. Während mit zunehmendem Lappengewicht das Risiko für Fettgewebsnekrosen steigt, kann dies durch eine steigende Anzahl Perforatoren kompensiert werden [27]. Da die Anzahl an Perforatoren in näherungsweiser Annahme in MS-TRAM Lappenplastiken erhöht sein sollte, ist es nicht verwunderlich, dass in einer Übersicht der aktuellen Literatur die Lappenverlustrate für MS-TRAM Lappen für 0,3-3,6\%, die für DIEP-Lappen für 5-6\% angegeben wird [28]. Allerdings zeigen diese Arbeiten auch die Abhängigkeit der DIEP-Komplikationsrate vom technischen Vorgehen in Bezug auf Perforatorenauswahl, Anzahl der eingeschlossenen Perforatoren und Lappengewicht. Diese Faktoren sind durch die Erfahrung des jeweiligen Operateurs beeinflusst und finden in vergleichenden Analysen keine Berücksich-

\begin{tabular}{|l|l|l|}
\hline MS-TRAM & DIEP & Vorteilhafteres Verfahre \\
\hline $0,3-3,6 \%$ & $0,5-6 \%$ & MS-TRAM \\
\hline $4,6-12,9 \%$ & $5,9-29 \%$ & MS-TRAM \\
\hline $3-5 \%$ & $0-2 \%$ & Tendenziell DIEP besser \\
\hline $6-21 \%$ & $5-6 \%$ & DIEP \\
\hline $1,9-5 \%$ & $0-2 \%$ & Tendenziell DIEP besser \\
\hline $6-21 \%$ & $0-5 \%$ & DIEP \\
\hline
\end{tabular}

tigung. Relevant ist in diesem Zusammenhang auch die Entwicklung neuer technischer Verfahren, die allerdings noch nicht in der Breite vorhanden sind. Durch intraoperative Indocyaningrün-(ICG)Angiographie lässt sich das tatsächlich optimal perfundierte Gewebe des Transplantates exakt bestimmen und erlaubt die individuelle patientenspezifische Optimierung des Lappendesigns [29]. Dadurch ist analog zu anderen freien Gewebetransplantationen mit einer weiteren Optimierung der Erfolgsrate bzw. einer Reduktion der oben genannten Probleme zu rechnen [30].

Diese Daten suggerieren somit, dass der DIEP-Lappen mehr Variablen bietet, die erfahrungsabhängig mit einer potentiell größeren Komplikationsrate einhergehen können. Insofern ist es wichtig die Vorteile der Hebedefektmorbidität gegenüber dem evtl. erhöhten Risiko zu analysieren und abzuwägen.

\section{Aktuelle Datenlage in der Literatur bzgl. Hebedefektmorbidität}

Gegensätzlich zur postulierten Durchblutungssicherheit der TRAMLappenplastik verhält es sich mit der Hebedefektmorbidität. Hier kann es zu Schmerzen und durch Schwächung der Bauchwand zu Instabilitäten inklusive dem sogenannten Bulging bis hin zu echten Hernien kommen. Das Ausmaß der Schwächung der Bauchwand hängt dabei von mehreren Faktoren ab, u. a. der Größe des entfernten Muskelanteils, der Größe des Fasziendefektes, der erhaltenen Innervation, der Verschlusstechnik sowie von individuellen anatomischen Gegebenheiten [12, 31, 32]. Es wird angenommen, dass die DIEP-Lappenplastik eine geringere Schädigung oder Schwächung der Bauchwand bewirkt.

Neben den o. g. funktionellen Faktoren spielen auch postoperative Schmerzen und Konturunregelmäßigkeiten bei der Bewertung der Hebedefektmorbidität eine Rolle. Bezüglich der postoperativen Schmerzen der Bauchwand scheinen diese bei DIEP- Lappenplastiken je nach Studie teilweise deutlich reduziert zu sein im Vergleich zu MS-TRAM-Lappenplastiken [33, 34], wohingegen keine wesentlichen Unterschiede bzgl. der postoperativen Bauchwandkontur zu vermerken sind [31, 35, 36].

Allerdings schränkt bei dem Versuch der systematischen Aufarbeitung dieser Annahme die Qualität der Berichterstattung und die Nachvollziehbarkeit der Nomenklatur der durchgeführten Lappenplastik die Auswertbarkeit von vergleichenden Studien bzgl. der Hebedefektmorbidität von MS-TRAM und DIEP- Lappenplas- 

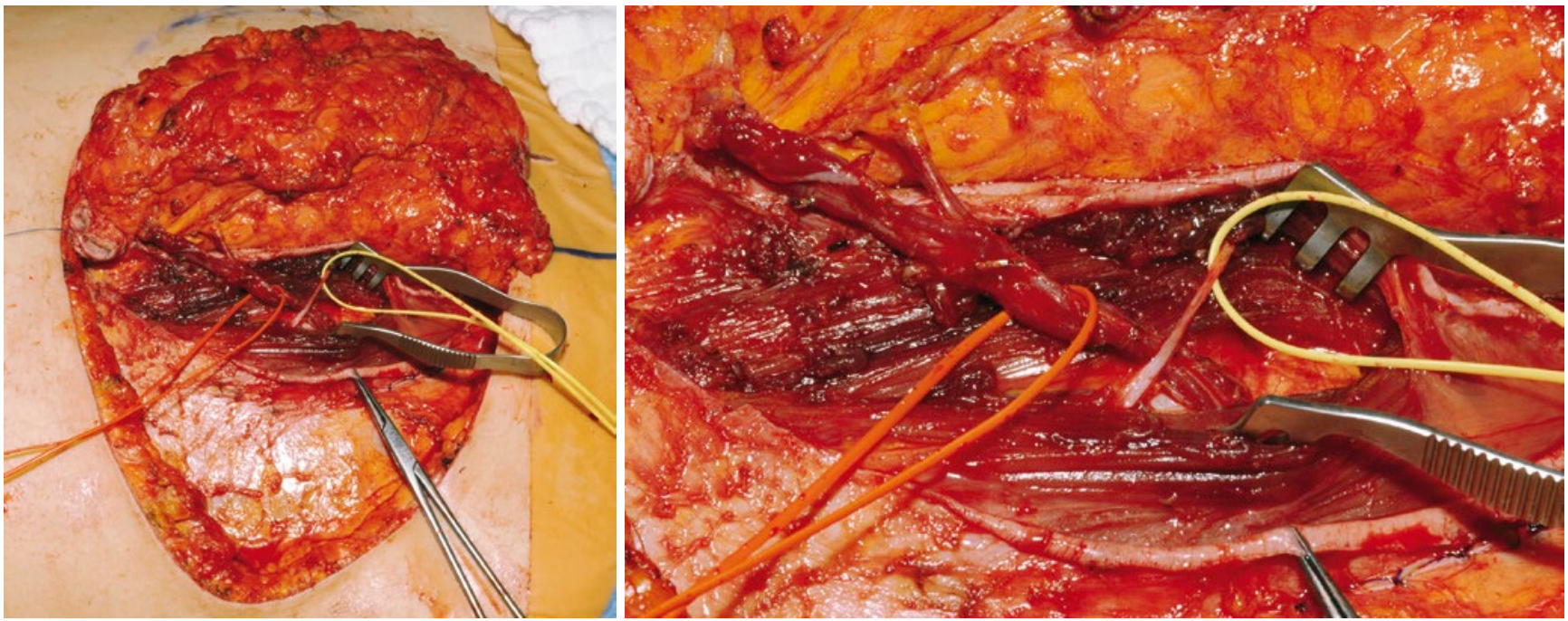

Abb. 2 Darstellung der Hebung der Lappenplastik von lateral unter Erhalt der Faszie und Freipräparation des Lappenstiels (roter Loop) mit zwei Perforatoren unter Schonung eines segmentalen intercostalen Nerven (gelber Loop).

tiken ein. Eine Metaanalyse der Datenlage identifizierte 200937 vergleichbare Studien die ein um etwa $50 \%$ reduziertes Risiko der Bauchwandschwäche (Bulging oder Hernie) nach einer DIEP-Lappenplastik im Vergleich zu TRAM-Lappenplastiken zeigte, bei einer gleichzeitig erhöhten Lappen-assoziierten Komplikationsrate in der DIEP-Gruppe [37]. Dies bestätigte auch eine Metaanalyse, die von 2154 gescreenten Publikationen mit schärferen Kriterien lediglich 5 Studien auswerten konnten [38]. Auch diese Analyse zeigte einen Trend hinsichtlich einer geringeren Hebedefektmorbidität der DIEP-Lappenplastik im Vergleich zur MS-TRAM Lappenplastik. Generell scheint das Risiko der Hernienbildung beim unilateralen MS-TRAM erhöht gegenüber der DIEP- Lappenplastik, auch wenn nicht alle Studien ein Signifikanzniveau von < 0,05 er reichen [39], $[7,13,31,35]$. Wichtig scheint dabei weniger die absolute Größe des in der TRAM-Lappenplastik eingeschlossenen Muskelsegmentes, als die Größe des Fasziendefektes und der Erhalt der Innervation der Restmuskulatur zu sein, was sich nur unvollständig mit Hilfe der MS-TRAM-Klassifikation retrospektiv analysieren lässt. Die Schwäche der o.g. Metaanalysen liegt in der Qualität der eingeschlossenen Originalarbeiten. Letztlich wird das Problem der retrospektiven Analyse exemplarisch dargestellt an dem Einschluss von Originalarbeiten die MS-0-TRAM-Lappenplastiken mit DIEPLappenplastiken vergleichen, und somit zu einer Verzerrung der Ergebnisse führen können [31].

Mit der Einschränkung des retrospektiven Studiencharakters ist die aktuelle Literatur jedoch relativ eindeutig im Bezug auf die Hebedefektmorbidität dieser Lappenplastiken bei bilateralen Rekonstruktionen. Hier ist das Risiko bei bilateralen MS-TRAM Lappenplastiken im Vergleich zu bilateralen DIEP-Lappenplastiken deutlich erhöht [12, 38] ( Tab. 2).

Eine aktuelle Arbeit von Uda et al. [40] zeigte sowohl nach MS-2TRAM als auch nach DIEP-Lappenplastiken eine temporäre Bauchwandschwäche, die sich schneller nach einer DIEP-Lappenplastik erholte. Dies wurde vergleichend in 42 Patienten mit unilateraler DIEP-Lappenplastiken und 36 Patienten mit MS-2-TRAM Lappenplastiken untersucht mittels Messung der isokinetischen Kraftentwicklung bei Anspannung der Bauchmuskulatur prä- und post- operativ. Langfristig (6 Monate postoperativ) waren die Ergebnisse bzgl. Kraftentwicklung der Bauchwand gleichwertig. Die Autoren wiesen diesbezüglich ausdrücklich auf die Schonung der Innervation der Rektusmuskulatur bei Hebung beider Lappentypen hin, die sicherlich die vergleichbaren Ergebnisse erklären kann. In einer großen retrospektiven „single-center“ Analyse konnte von Chang und Mitarbeitern [32] gezeigt werden, dass durch Schonung des Bauchmuskels bei DIEP-Lappenplastiken die Hebedefektmorbidität reduziert werden kann. Allerdings war dieser Effekt nicht mehr signifikant, wenn ein Netz zur Stabilisierung des Fasziendefektes eingebracht wurde. Dies konnte auch von anderen Arbeitsgruppen bestätigt werden [41]. Diese Arbeiten belegen, dass das technische Vorgehen, unabhängig von der gewählten Lappenplastik, nicht nur bei der Lappenhebung, sondern auch beim Verschluss der Hebestelle wesentlich die Bauchwandstabilität beeinflusst. Dies wiederum verdeutlicht die Schwierigkeit in der Interpretation verschiedener Studien, da die Operateur-abhängigen individuellen Unterschiede nicht zu unterschätzen sind. Den meisten Arbeiten ist der retrospektive Charakter der Studien gemein, der das Evidenzniveau natürlich nachhaltig beeinträchtigt.

\section{Konsensus der Arbeitsgruppe}

Auf Boden der dargestellten Problematik besteht ein Expertenkonsensus, dass ein vermeintlich hochwertigerer Lappen in der MS Klassifikation nicht immer mit geringerer Hebedefektmorbidität korreliert. Aus den in der Literatur beschriebenen Vorteilen der jeweiligen Verfahren muss der erfahrene Chirurg einen Kompromiss aus Perfusionssicherheit und möglichst geringer Hebedefektmorbidität finden. Allerdings wird hier auch in Zukunft die definitive Evidenzfindung schwierig, da erhebliche Probleme in der Standardisierung, der Klassifikation und der OP Technik gesehen werden. Die Präparation eines DIEPs kann sich von Operateur zu Operateur wesentlich unterscheiden und die Vergleichbarkeit und damit retrospektive Analysen verzerren bis zu unmöglich machen. Dennoch sollten generelle Prinzipien verinnerlicht werden und die Schaffung von Fasziendefekten sowie die Durchtrennung von Ner- 


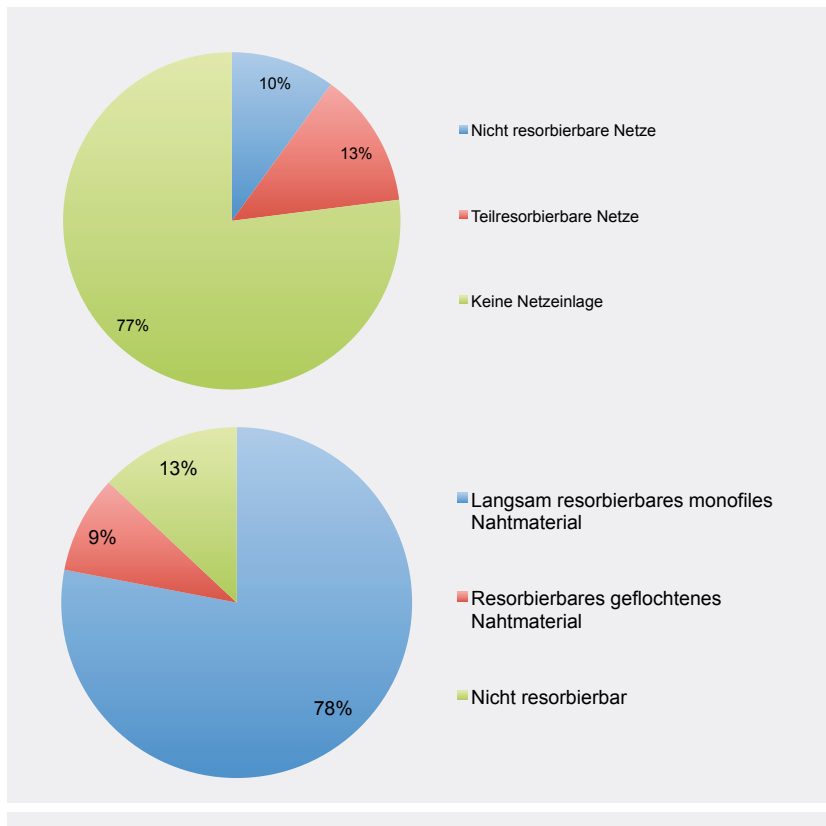

-Abb. 3 Meinungsbild der Expertenrunde. Darstellung der Einbringung eines Netzes zur Bauchwandrekonstruktion (oben) und der Wahl des Nahtmaterials zum Faszienverschluss (unten). Die Mehrheit der Teilnehmer bevorzugt langsam resorbierbares monofiles Nahtmaterial zum Faszienverschluss (78\%) und bringt kein alloplastisches Material ein (77\%).

ven vermieden werden. Die Operationsstrategie und Präparationsstrategie sollte sich daher in Sorgfalt und Gewebeschonung zwischen TRAM und DIEP nicht unterscheiden ( $>$ Abb. 2). Die initialen Schritte der Lappenhebung und Perforatordarstellung von lateral sowie Schonung der lateralen Muskelanteile sollten identisch und das Ziel sein. Allerdings besteht ein Expertenkonsensus, dass die Mitnahme von kleineren Muskelsegmenten unter beachten dieser Vorgaben wahrscheinlich irrelevant ist. Daher scheint der freie muskelsparende TRAM per se nicht schlechter zu sein als der DIEP. Die Verletzung einzelner segmentaler Nervenäste lässt sich gelegentlich nicht vermeiden und kann toleriert werden. Bei Verletzung von mehr als einem segmentalen intercostalen Nervenast sollte die Nervennaht erwogen werden. Hier ist besonders auf die dominanten Nervenäste hinzuweisen, die etwa auf Höhe der Linea arcuata in den Muskel eintreten [42, 43].

Die Mitnahme eines kompletten Rektus-Segmentes in voller Breite wird als „out of date“ angesehen. Ein besonderes Augenmerk sollte auf die Technik des Faszienverschlusses gelegt werden. Hier sei insbesondere auf die anatomische Problematik der Hebung eines Muskelsegmentes unterhalb der Linea arcuata hingewiesen. Hier kommt es auf Grund des Fehlens des hinteren Blattes der Rektusscheide zu vermehrtem Bulging und die Hebung eines kleinen Muskelsegmentes sollte wenn möglich oberhalb davon erfolgen. Die aktuelle Literatur scheint Vorteile in der Reduktion der Hebedefektmorbidität durch das Einbringen von alloplastischen Netzen zu sehen [7, 12, 14, 32, 41]. Insbesondere bei bilateralen Rekonstruktionen kann die Hebedefektmorbidität der TRAM Lappenplastik damit reduziert werden [41]. Dennoch verwendet die Mehrheit der Teilnehmer des Expertengremiums keine alloplastischen Netze zur Rekonstruktion der Bauchwand. Die Mehrheit be- vorzugt eine fortlaufende Fasziennaht mit langsam resorbierbarem Nahtmaterial ( $\triangleright$ Abb. 3 ).

\section{Zusammenfassung und DAM Konsensus-Statements}

Zusammenfassend besteht auf Grundlage der aktuellen Literatur und der persönlichen und institutionellen Erfahrung in der Expertenrunde Einigkeit über folgende Punkte:

- Der freie MS-TRAM und DIEP ist dem gestielten TRAM zur Brustrekonstruktion überlegen.

- Bezüglich der freien Lappenplastiken sollte das sicherste Verfahren mit der möglichst geringsten Hebedefektmorbidität gewählt werden. Bezüglich der Durchblutungssicherheit scheint der MS-TRAM dem DIEP-Lappen im Zweifelsfall überlegen zu sein.

- Der unilaterale MS-TRAM muss mit den richtigen Präparationstechniken und adäquatem Hebestellenverschluss nach aktueller Datenlage dem unilateralen DIEP in punkto Hebedefektmorbidität als gleichwertig angesehen werden.

- In diesem Spannungsfeld und auf Boden der gegenwärtigen Literaturlage lohnt es sich nicht, bei unsicheren Perforatoren den DIEP-Lappen zu erzwingen zu Gunsten einer vermeintlich geringeren Hebedefektmorbidität.

- Bei bilateralen Brustrekonstruktionen sollte im Interesse des Hebedefektes nach Möglichkeit zumindest bei einer Seite eine DIEP Lappenplastik gehoben werden.

Der Expertenkonsensus beruht auf der aktuellen Literaturlage, die kaum über das Evidenzlevel 3 hinausgeht. Auch wenn die retrospektiven und teilweise prospektiven Arbeiten ein relativ klares Bild liefern, ist dies jedoch eine klare Einschränkung, die von Befürwortern anderer Rekonstruktionstechniken negativ ausgelegt werden könnten. Die Problematik dieser Limitation zeigt sich unter anderem auch in einem aktuellen Konsensuspapier der American Society of Plastic Surgeons (ASPS), dass auf Grund dieser Limitation keinen klaren Vorteil des DIEP Lappens gegenüber dem gestielten TRAM feststellen konnte [44]. Diese Sichtweise ist nur schwierig zu entkräften und eine prospektiv-randomisierte Studie ist bei der vorliegenden Datenlage ethisch nur schwer begründbar, da die retrospektive Datenlage sehr deutlich die Vorteile der freien Lappenplastiken gegenüber dem gestielten TRAM in Bezug auf Reduktion der Hebedefektmorbidität [3] und Lappen-assoziierten Komplikationen wie Fettegewebsnekrosen und Lappenteilverluste [4] belegt. Dies gilt auch für den Vergleich der freien TRAM Lappenplastiken mit den hier beschriebenen muskelsparenden Variationen [7], auch wenn hier die Einschränkungen der häufig unklaren Nomenklatur in der Literatur, die eingangs bereits erläutert wurde die direkt vergleichende Analyse erschwert. Unstrittig stellen jedoch sowohl die DIEP- als auch die MS-TRAM Lappenplastik in den Händen eines erfahrenen Operateurs sichere Verfahren zur uni- und bilateralen autologen Brustrekonstruktion dar. Beide Techniken weisen eine geringe Komplikationsrate auf und führen zu einer geringen Hebedefektmorbidität. 


\section{Interessenkonflikt}

Die Autoren geben an, dass kein Interessenkonflikt besteht.

\section{Autorinnen/Autoren}

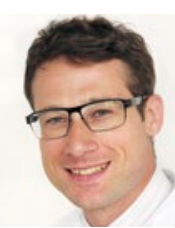

\section{Prof. Steffen Eisenhardt}

ist Universitätsprofessor für Klinische und Experimentelle Mikrochirurgie an der Medizinischen Fakultät der Albert-Ludwigs Universität Freiburg. Er leitet die Sektion für Rekonstruktive Mikrochirurgie der Klinik für Plastische und Handchirurgie am Universitätsklinikum Freiburg. Seine klinischen Schwerpunkte liegen in der interdisziplinären rekonstruktiven Mikrochirurgie und der funktionellen Kopf/Hals Chirurgie. Als Heisenberg-Professor der Deutschen Forschungsgemeinschaft (DFG) liegen seine wissenschaftlichen Schwerpunkte auf der Erarbeitung der Rolle der angeborenen Immunantwort in akuten und chronischen Entzündungs- und Abstoßungsreaktionen.

\section{Literatur}

[1] Hartrampf CR, Scheflan M, Black PW. Breast reconstruction with a transverse abdominal island flap. Plastic and reconstructive surgery 1982; 69(2): 216-25

[2] Holmstrom $\mathrm{H}$. The free abdominoplasty flap and its use in breast reconstruction. An experimental study and clinical case report. Scand J Plast Reconstr Surg 1979; 13(3): 423-27

[3] Knox AD, Ho AL, Leung L et al. Comparison of Outcomes following Autologous Breast Reconstruction Using the DIEP and Pedicled TRAM Flaps: A 12-Year Clinical Retrospective Study and Literature Review. Plastic and reconstructive surgery 2016; 138(1): 16-28

[4] Jeong W, Lee S, Kim J. Meta-analysis of flap perfusion and donor site complications for breast reconstruction using pedicled versus free TRAM and DIEP flaps. Breast 2017; 38: 45-51

[5] Koshima I, Soeda S. Inferior epigastric artery skin flaps without rectus abdominis muscle. Br J Plast Surg 1989; 42(6): 645-8

[6] Allen RJ, Treece P. Deep inferior epigastric perforator flap for breast reconstruction. Annals of plastic surgery 1994; 32(1): 32-8

[7] Nahabedian MY, Momen B, Galdino G et al. Breast Reconstruction with the free TRAM or DIEP flap: patient selection, choice of flap, and outcome. Plastic and reconstructive surgery 2002; 110(2): 466-75; discussion 76-7

[8] Keller A. Fat necrosis in free rectus abdominis and deep inferior epigastric perforator flaps. Plastic and reconstructive surgery 2001; 107(6): 1611-2

[9] Selber JC, Fosnot J, Nelson ] et al. A prospective study comparing the functional impact of SIEA, DIEP, and muscle-sparing free TRAM flaps on the abdominal wall: Part II. Bilateral reconstruction. Plastic and reconstructive surgery 2010; 126(5): 1438-53

[10] Selber JC, Nelson J, Fosnot J et al. A prospective study comparing the functional impact of SIEA, DIEP, and muscle-sparing free TRAM flaps on the abdominal wall: part I. unilateral reconstruction. Plastic and reconstructive surgery 2010; 126(4): 1142-53

[11] Selber JC, Serletti JM. The deep inferior epigastric perforator flap: myth and reality. Plastic and reconstructive surgery 2010; 125(1): 50-8

[12] Nahabedian MY, Tsangaris T, Momen B. Breast reconstruction with the DIEP flap or the muscle-sparing (MS-2) free TRAM flap: is there a difference? Plastic and reconstructive surgery 2005; 115(2): 436-44; discussion 45-6

[13] Chen CM, Halvorson EG, Disa J] et al. Immediate postoperative complications in DIEP versus free/muscle-sparing TRAM flaps. Plastic and reconstructive surgery 2007; 120(6): 1477-82

[14] Nelson JA, Guo Y, Sonnad SS et al. A Comparison between DIEP and muscle-sparing free TRAM flaps in breast reconstruction: a single surgeon's recent experience. Plastic and reconstructive surgery 2010; 126(5): 1428-35

[15] Kroll SS. Fat necrosis in free transverse rectus abdominis myocutaneous and deep inferior epigastric perforator flaps. Plastic and reconstructive surgery 2000; 106(3): 576-83

[16] Schusterman MA, Kroll SS, Miller MJ et al. The free transverse rectus abdominis musculocutaneous flap for breast reconstruction: one center's experience with 211 consecutive cases. Annals of plastic surgery 1994; 32(3): 234-41; discussion 41-2

[17] Mehrara B], Santoro TD, Arcilla E et al. Complications after microvascular breast reconstruction: experience with 1195 flaps. Plastic and reconstructive surgery 2006; 118(5): 1100-9; discussion 10-1

[18] Damen TH, Morritt AN, Zhong T et al. Improving outcomes in microsurgical breast reconstruction: lessons learnt from 406 consecutive DIEP/TRAM flaps performed by a single surgeon. J Plast Reconstr Aesthet Surg 2013; 66(8): 1032-8

[19] Gill PS, Hunt JP, Guerra AB et al. A 10-year retrospective review of 758 DIEP flaps for breast reconstruction. Plastic and reconstructive surgery 2004; 113(4): 1153-60

[20] Hamdi M, Weiler-Mithoff EM, Webster MH. Deep inferior epigastric perforator flap in breast reconstruction: experience with the first 50 flaps. Plastic and reconstructive surgery 1999; 103(1): 86-95

[21] Blondeel PN. One hundred free DIEP flap breast reconstructions: a personal experience. Br J Plast Surg 1999; 52(2): 104-11

[22] Scheer AS, Novak CB, Neligan PC et al. Complications associated with breast reconstruction using a perforator flap compared with a free TRAM flap. Annals of plastic surgery 2006; 56(4): 355-8

[23] Giunta RE, Horch RE, Prantl L et al. [Consensus of the Deutsche Gesellschaft der Plastischen, Rekonstruktiven und Asthetischen Chirurgen (DGPRAC) on Autologous Fat Grafting]. Handchir Mikrochir Plast Chir 2016; 48(6): 337-9

[24] Prantl L, Rennekampff HO, Giunta RE et al. [Current Perceptions of Lipofilling on the Basis of the New Guideline on "Autologous Fat Grafting"]. Handchir Mikrochir Plast Chir 2016; 48(6): 330-6

[25] Kamali P, Lee M, Becherer BE et al. Medial Row Perforators Are Associated with Higher Rates of Fat Necrosis in Bilateral DIEP Flap Breast Reconstruction. Plastic and reconstructive surgery 2017; 140(1): 19-24

[26] Baumann DP, Lin HY, Chevray PM. Perforator number predicts fat necrosis in a prospective analysis of breast reconstruction with free TRAM, DIEP, and SIEA flaps. Plastic and reconstructive surgery 2010; 125(5): 1335-41

[27] Mulvey CL, Cooney CM, Daily FF et al. Increased Flap Weight and Decreased Perforator Number Predict Fat Necrosis in DIEP Breast Reconstruction. Plastic and reconstructive surgery Global open 2013; 1(2): 1-7

[28] Butler PD, Wu LC. Abdominal perforator vs. muscle sparing flaps for breast reconstruction. Gland surgery 2015; 4(3): 212-21

[29] Ludolph I, Arkudas A, Schmitz M et al. Cracking the perfusion code?: Laser-assisted Indocyanine Green angiography and combined laser Doppler spectrophotometry for intraoperative evaluation of tissue perfusion in autologous breast reconstruction with DIEP or ms-TRAM flaps. J Plast Reconstr Aesthet Surg 2016; 69(10): 1382-8

[30] Buehrer G, Taeger CD, Ludolph I et al. Intraoperative flap design using ICG monitoring of a conjoined fabricated anterolateral thigh/tensor fasciae latae perforator flap in a case of extensive soft tissue reconstruction at the lower extremity. Microsurgery 2016; 36(8): 684-8 
[31] Blondeel N, Vanderstraeten GG, Monstrey SJ et al. The donor site morbidity of free DIEP flaps and free TRAM flaps for breast reconstruction. Br J Plast Surg 1997; 50(5): 322-30

[32] Chang El, Chang El, Soto-Miranda MA et al. Comprehensive analysis of donor-site morbidity in abdominally based free flap breast reconstruction. Plastic and reconstructive surgery 2013; 132(6): 1383-91

[33] Kroll SS, Sharma S, Koutz C et al. Postoperative morphine requirements of free TRAM and DIEP flaps. Plastic and reconstructive surgery 2001; 107(2): 338-41

[34] Wu LC, Bajaj A, Chang DW et al. Comparison of donor-site morbidity of SIEA, DIEP, and muscle-sparing TRAM flaps for breast reconstruction. Plastic and reconstructive surgery 2008; 122(3): 702-9

[35] Nahabedian MY, Dooley W, Singh N et al. Contour abnormalities of the abdomen after breast reconstruction with abdominal flaps: the role of muscle preservation. Plastic and reconstructive surgery 2002; 109(1): 91-101

[36] Nahabedian MY, Manson PN. Contour abnormalities of the abdomen after transverse rectus abdominis muscle flap breast reconstruction: a multifactorial analysis. Plastic and reconstructive surgery 2002; 109(1): 81-7; discussion 8-90

[37] Man LX, Selber JC, Serletti JM. Abdominal wall following free TRAM or DIEP flap reconstruction: a meta-analysis and critical review. Plastic and reconstructive surgery 2009; 124(3): 752-64

[38] Egeberg A, Rasmussen MK, Sorensen JA. Comparing the donor-site morbidity using DIEP, SIEA or MS-TRAM flaps for breast reconstructive surgery: a meta-analysis. J Plast Reconstr Aesthet Surg 2012; 65(11): 1474-80

[39] Bajaj AK, Chevray PM, Chang DW. Comparison of donor-site complications and functional outcomes in free muscle-sparing TRAM flap and free DIEP flap breast reconstruction. Plastic and reconstructive surgery 2006; 117(3): 737-46; discussion 47-50

[40] Uda H, Kamochi H, Sarukawa S et al. Clinical and Quantitative Isokinetic Comparison of Abdominal Morbidity and Dynamics following DIEP versus Muscle-Sparing Free TRAM Flap Breast Reconstruction. Plastic and reconstructive surgery 2017; 140(6): 1101-9
[41] Wan DC, Tseng CY, Anderson-Dam J et al. Inclusion of mesh in donorsite repair of free TRAM and muscle-sparing free TRAM flaps yields rates of abdominal complications comparable to those of DIEP flap reconstruction. Plastic and reconstructive surgery 2010; 126(2): 367-74

[42] Rozen WM, Ashton MW, Kiil B] et al. Avoiding denervation of rectus abdominis in DIEP flap harvest II: an intraoperative assessment of the nerves to rectus. Plastic and reconstructive surgery 2008; 122(5): $1321-5$

[43] Rozen WM, Ashton MW, Murray AC et al. Avoiding denervation of rectus abdominis in DIEP flap harvest: the importance of medial row perforators. Plastic and reconstructive surgery 2008; 122(3): 710-6

[44] Lee BT, Agarwal JP, Ascherman JA et al. Evidence-Based Clinical Practice Guideline: Autologous Breast Reconstruction with DIEP or Pedicled TRAM Abdominal Flaps. Plastic and reconstructive surgery 2017; 140(5): 651e-64e

\section{ERRATUM}

In die vorliegende Arbeit haben sich leider ein paar Fehler in den englischen Titel reingeschlichen.

Der korrekte englische Titel lautet: Breast reconstruction with the free TRAM or DIEP flap - What is the current standard?

Consensus Statement of the German Speaking Working Group for Microsurgery of the Peripheral Nerves and Vessels

Wir bitten diese Fehler zu entschuldigen. 